\title{
Antibacterial, anti-swarming and anti-biofilm formation activities of Chamaemelum nobile against Pseudomonas aeruginosa
}

\author{
Hossein Kazemian ${ }^{[1],[2], ~ S o b h a n ~ G h a f o u r i a n ~}{ }^{[1]}$, Hamid Heidari[ ${ }^{[3]}$, Pouya Amiri ${ }^{[4]}$, \\ Jalil Kardan Yamchi ${ }^{[5]}$, Aref Shavalipour ${ }^{[4]}$, Hamidreza Houri ${ }^{[4]}$, Abbas Maleki ${ }^{[1]}$ \\ and Nourkhoda Sadeghifard ${ }^{[1]}$
}

[1]. Clinical Microbiology Research Center, Ilam University of Medical Sciences, Ilam, Iran. [2]. Department of Medical Microbiology, School of Medicine, Tehran University of Medical Sciences, Tehran, Iran. [3]. Department of Bacteriology and Virology, School of Medicine, Shiraz University of Medical Sciences, Shiraz, Iran. [4]. Department of Medical Microbiology, Shahid Beheshti University of Medical Sciences, Tehran, Iran. [5]. Department of Pathobiology, Division of Microbiology, School of Public Health, Tehran University of Medical Sciences, Tehran, Iran.

\begin{abstract}
Introduction: Chamomile (Chamaemelum nobile) is widely used throughout the world, and has anti-inflammatory, deodorant, bacteriostatic, antimicrobial, carminative, sedative, antiseptic, anti-catarrhal, and spasmolytic properties. Because of the increasing incidence of drug-resistant bacteria, the development of natural antibacterial sources such as medical herbs for the treatment of infectious diseases is necessary. Extracts from different plant parts such as the leaves, flowers, fruit, and bark of Combretum albiflorum, Laurus nobilis, and Sonchus oleraceus were found to possess anti-quorum sensing (QS) activities. In this study, we evaluated the effect of $C$. nobile against Pseudomonas aeruginosa biofilm formation. Methods: The P. aeruginosa samples were isolated from patients with different types of infection, including wound infection, septicemia, and urinary tract infection. The flowers of $C$. nobile were dried and the extract was removed using a rotary device and then dissolved in dimethyl sulfoxide at $\mathrm{pH}$ 7.4. The microdilution method was used to evaluate the minimum inhibitory concentration (MIC) of this extract on $P$. aeruginosa, and biofilm inhibition was assayed. Results: Eighty percent of the isolated samples (16/20) could form a biofilm, and most of these were isolated from wound infections. The biofilm inhibitory concentration of the C. nobile extract was $6.25-25 \mathrm{mg} / \mathrm{ml}$, whereas the MIC was $12.5-50 \mathrm{mg} / \mathrm{ml}$. Conclusions: The anti-QS property of C. nobile may play an important role in its antibacterial activity, thus offering an additional strategy in the fight against bacterial infections. However, molecular investigation is required to explore the exact mechanisms of the antibacterial action and functions of this phytocompound.
\end{abstract}

Keywords: Anti-quorum sensing (QS). Antibacterial. Chamaemelum nobile. Biofilm. Pseudomonas aeruginosa.

\section{INTRODUCTION}

The $21^{\text {st }}$ century has seen a reversion from chemical drugs to increased interest in the utilization of natural products for medicinal purposes, particularly plants. Accordingly, there have been many developments with respect to investigations of medical herbs, and this field is expected to expand in the future. The use of dried herb extracts (total extracts) instead of purified herb materials can increase the efficiency of an herb's medical effects with less side effects, because of the diluted concentration of the plant material. Such extracts come in various forms such

Corresponding author: Dr. Nourkhoda Sadeghifard. Department of Microbiology/Clinical Microbiology Research Center/Ilam University of Medical Sciences. Banganjab st., Pajouhesh Blvd., Ilam, Iran.

Phone: 98918 794-8939; Fax: 98841 222-7101

e-mail: sadeghifard-n@medilam.ac.ir

Received 5 March 2015

Accepted 10 June 2015 as tablets, capsules, ointments, syrup, and others, which are suitable for contemporary lifestyle. Because of the increasing demand for medical herbs for the treatment of various ailments and conditions, more research in this field is essential ${ }^{(1)(2)(3)}$.

Several plants with antibacterial effects are commonly used in medicine, such as chamomile (Chamaemelum nobile), which is a small aromatic plant that is a traditional medical herb with anti-inflammatory, antimicrobial, and antiseptic properties. It has long been used for the treatment of several conditions such as malaria, peptic ulcers, wound healing, prevention of gangrene, and others ${ }^{(4)}$.

Many host-associated bacteria use chemical signals to monitor their own population density and to control the expression of specific genes in response to population density. This type of gene regulation is termed quorum sensing $(\mathrm{QS})^{(5)}$, which is a general phenomenon identified in many gram-negative and gram-positive bacteria ${ }^{(6)(7)}$. Many gram-negative bacteria employ $\mathrm{N}$-acyl homoserine lactones (AHLs) as QS signaling compounds, which vary among bacteria with respect to length and the specific substitutions of their acyl side chains. The signaling molecule 
is synthesized by a LuxI-type synthase and binds to a cognate LuxR-type transcriptional activator protein to regulate the expression of target genes. At low cell density, the signaling compound is synthesized at a low basal level and is thought to diffuse into the surrounding medium where it becomes diluted. However, during growth, the AHL concentration in the medium rises to a critical threshold. At this concentration, AHL becomes activated by binding to its cognate receptor, and consequently stimulates or represses the transcription of target genes ${ }^{(8)}$. Such a QS process regulates important bacterial activities such as virulence gene expression and biofilm formation.

Biofilms are a highly structured, surface-attached community of cells enclosed in a self-produced polymeric matrix. In laboratory-based systems, Pseudomonas aeruginosa forms a biofilm (several hundred micrometers in thickness) with tower- and mushroom-shaped microcolonies intervened by water channels and void spaces ${ }^{(9)}$. Pseudomonas aeruginosa, a gram-negative opportunistic human pathogen, is responsible for persistent and often incurable infections in immunocompromised people or patients with cystic fibrosis ${ }^{(10)}$.

The most recent strategies aimed at discovering new inhibitors that are able to disrupt the different steps of the QS system have focused on targeting signal production, signal molecules, and signal receptors ${ }^{(11)}$. To overcome the biofilm formation problem of antibiotic-resistant bacteria, an antipathogenic approach has been recently considered as a viable alternative, and inhibition of QS is a particularly attractive target. Thus, researchers have focused on plant-derived antimicrobials as an alternative to the more ineffective antibiotics ${ }^{(12)}$.

Therefore, the development of natural antibacterial elements with other sources such as medical herbs is essential for novel treatments of infectious diseases ${ }^{(13)}$. Considering its reported curative properties, we decided to evaluate the effect of $C$. nobile against biofilm formation of $P$. aeruginosa.

\section{METHODS}

\section{Samples}

Samples were isolated from different infection sources, including wound infection, septicemia, and urinary tract infections, from 20 patients of Immam Khomini Hospital in Ilam City. We selected the strains with multidrug-resistant features (i.e., resistant to cloxacillin, cotrimoxazole, cephazolin, carbenicillin, piperacillin, and ceftazidime). Isolates from patients were cultured in tripticase soy broth (TSB) and were stored in $-70^{\circ} \mathrm{C}$, and then freshly sub-cultured on brain heart infusion (BHI) agar prior to each assay.

\section{Herbal extraction}

Chamaemelum nobile plants were collected from a wildgrowing population at the full-flowering stage, from the mountains of the Ilam province of Iran. The flowers were dried out and the extracts were removed using a rotary device. The acquired suspension was then concentrated via a vacuum distillation process. To make the suspension, the extract was dissolved in dimethyl sulfoxide at $\mathrm{pH}$ 7.4. The antibacterial properties of the extracts were initially determined using the broth microdilution method, and the dry weight of the extracts was calculated per milliliter.

\section{Determination of minimum inhibitory concentration and minimum bactericidal concentration}

The minimum inhibitory concentration (MIC) values of plant extracts were determined by the broth microdilution method using 96-well microtiter plates. Serial two-fold dilutions of the plant extracts were prepared by vortexing the extracts in Millipore water. The inoculum of microorganisms was prepared in Mueller-Hinton Broth (Himedia, India), and the turbidity was adjusted to $0.5 \mathrm{McF}$ arland and diluted to obtain a final turbidity in wells of approximately $1 \times 10^{6}$ colony-forming units $(\mathrm{CFU}) / \mathrm{ml}$. Fifty micro liters of plant extract solution and $150 \mu \mathrm{l}$ of bacterial inoculum were placed into the wells of a microtiter plate and incubated at $37^{\circ} \mathrm{C}$ for $24 \mathrm{~h}$. The MIC is defined as the lowest concentration of antimicrobial agent that completely inhibits growth of the organism. Then, the minimum bactericidal concentration (MBC) value was determined using standard methods. In brief, the MBC is identified by determining the lowest concentration of antibacterial agent that reduces the viability of the initial bacterial inoculum by $\geq 99.9 \%^{(14)}$. Pseudomonas aeruginosa (PAO1) was used as a positive control.

\section{Data interpretation}

Absorbance values lower than the control cells indicated a reduction in the rate of cell proliferation. Conversely, a higher absorbance ratio indicated an increase in cell proliferation. Rarely, an increase in proliferation or morphological changes may be offset by cell death. The percent inhibition was calculated with the following formula: $\%$ inhibition $=1-$ $(\mathrm{OD}$ observed $/ \mathrm{OD}$ control $) \times 100$.

\section{Biofilm inhibition assay}

One colony of each P. aeruginosa isolate was used for inoculation in $5 \mathrm{ml}$ of BHI broth. The culture was incubated for $18 \mathrm{~h}$ at $37^{\circ} \mathrm{C}$ with aeration at $200 \mathrm{rpm}$. Following incubation, the number of cells in each culture was adjusted to $1.5 \times 10^{8}$ $\mathrm{CFU} / \mathrm{ml}(0.5 \mathrm{McF}$ arland), and $50 \mu \mathrm{l}$ of each bacterial suspension was transferred to eight wells of a 96-well microtiter plate. Eight replicates were used for each isolate in each biofilm assay. The biofilm-forming Staphylococcus epidermidis strain RP62A and PAO1, which are known to form fully established biofilms, were added to each plate as positive controls. Brain heart infusion broth was incorporated as a negative control. A 96-peg plate was then positioned in the wells of the microtiter plate, allowing the pegs to be submerged within the bacterial culture. The inoculated peg plate was transferred to a 96-well microtiter plate containing fresh BHI broth and incubated for $48 \mathrm{~h}$ at $37^{\circ} \mathrm{C}$ on a rocking platform, to allow mature biofilms to establish. Each biofilm assay was repeated twice independently.

Biofilm biomass was quantified using a modification of a methodology described by Mowat et al ${ }^{(15)}$. Following incubation, the peg plate was removed from the microtiter plate, rinsed twice in phosphate-buffered saline to remove loosely attached 
planktonic cells, and dried for $30 \mathrm{~min}$ at $37^{\circ} \mathrm{C}$. Each replicate peg was stained with filtered $0.5 \%(\mathrm{w} / \mathrm{v})$ crystal violet for $5 \mathrm{~min}$. Excess crystal violet was removed by gently washing the peg plate twice with distilled water. Replicate pegs were detached from the plate using needle-tipped pliers and added to $1 \mathrm{ml}$ of $70 \%$ ethanol to leach the crystal violet from the stained biofilms. The absorbance at $570 \mathrm{~nm}$ was measured using a microtiter plate reader. As the polystyrene pegs were suspended in the wells of the microtiter plate, any biomass that remained bound to the surface following the washing steps could be considered to be a genuine biofilm. The wells of the microtiter plate were not sampled for the presence of biofilm biomass, as this could have instead been a deposit of planktonic cells.

The capacity of each strain to form a biofilm was compared with the confluent biofilm control by analyzing the absorbance of the crystal violet stain obtained for each biofilm. This allowed for each isolate to be assigned a percentage value depending on the proportion of biofilm biomass it could establish after $48 \mathrm{~h}$ in comparison with the control (assuming a rate of $100 \%$ for the control). Eight replicate pegs were included for each isolate in each biofilm assay and the assay was carried out three times. Isolates were also divided into three groups depending on whether they formed fully established biofilms with $75 \%$ of the biomass of the positive control, moderately adherent biofilms with $25-75 \%$ of the control biomass, or weak biofilms with $25 \%$ of the biomass of the positive control.

To evaluate the effect of $C$. nobile in inhibiting biofilm formation, $100 \mu \mathrm{l}$ BHI broth was added to a 96-peg plate, and $50 \mu 1$ of the extract was transferred to each of eight wells and diluted. A 50- $\mu 1$ aliquot of each bacterial suspension (0.5 McFarland) that formed a biofilm (see above) was transferred to eight wells of a 96-well microtiter plate and incubated for $24 \mathrm{~h}$ at $37^{\circ} \mathrm{C}$.

\section{Swarming assay}

Fifty microliters of extract was mixed with $10 \mathrm{ml}$ of molten Mueller-Hinton agar medium and poured immediately over the surface of the plate as an overlay. The plate was pointinoculated with an overnight culture of PAO1 once the overlaid agar had solidified, and incubated at $37^{\circ} \mathrm{C}$ for 3 days. The extent of swarming was determined by measuring the area of the colony ${ }^{(16)}$. The experiment was performed in triplicate and repeated twice.

\section{RESULTS}

A total of 20 samples were collected from different sources of infection in patients that had been referred to Imam Khomini Hospital of Ilam, $16(80 \%)$ of the samples formed a biofilm. Of sixteen biofilm-forming $P$. aeruginosa, nine strains isolated from wound infections.

Chamaemelum nobile extract showed biofilm inhibitory activity in the tested concentration range of 1.6 to $100 \mathrm{mg} / \mathrm{ml}$. The effective concentrations against biofilm formation were $6.25-25 \mathrm{mg} / \mathrm{ml}$, whereas the MIC and MBC were $12.5-50 \mathrm{mg} / \mathrm{ml}$ and $25 \mathrm{mg} / 1$, respectively.
Flagella motility-dependent swarming is also regulated by QS. Therefore, a reduction in swarming area compared with the control plate would suggest the presence of anti-QS compounds. C. nobile, which shows anti-swarming activity, selectively and distinctly reduced the swarming area of PAO1, suggesting that these extracts could inhibit the swarming motility of PAO1 (Figure 1).

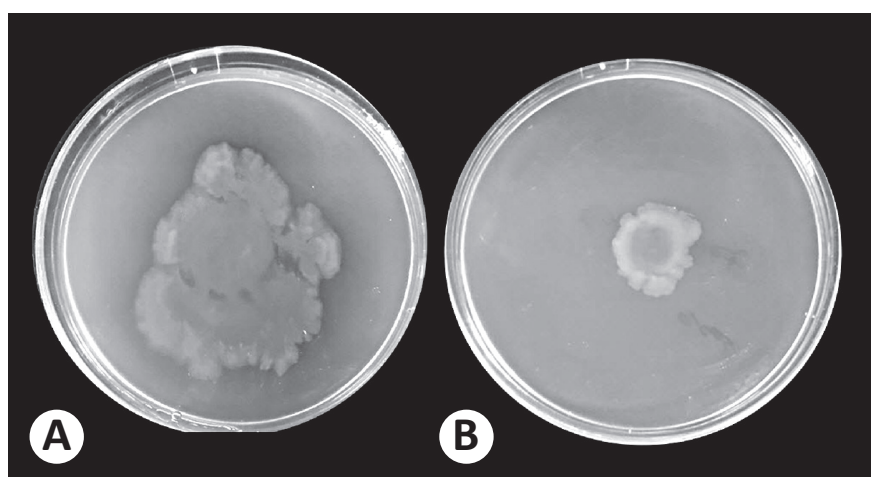

FIGURE 1 - Swarming inhibition of Pseudomonas aeruginosa PAO1 by Chamaemelum nobile extract. A) Pseudomonas aeruginosa PAO1 only (without extract). B) Pseudomonas aeruginosa PAO1 with Chamaemelum nobile extract. PAO1: standard strain of Pseudomonas aeruginosa.

\section{DISCUSSION}

Quorum sensing is a population density-dependent mechanism that is mediated through small signaling molecules called autoinducers by which bacteria regulate gene expression; in gramnegative bacteria, these autoinducers are AHLs. In some pathogenic bacteria, the QS system controls the expression of genes responsible for the production of virulence factors. Therefore, targeting QS has emerged as an alternative strategy for controlling bacterial virulence, and studies have identified both synthetic and natural compounds capable of disrupting QS, including some phytocompounds ${ }^{(17)}$.

The aim of this study was to evaluate the effect of chamomile extract on the biofilm formed by $P$. aeruginosa.

Chamaemelum nobile is widely used throughout the world, primarily as a sedative, anxiolytic, antispasmodic, and for the treatment of mild skin irritation and inflammation. Chamomile has anti-inflammatory, deodorant, bacteriostatic, antimicrobial, carminative, sedative, antiseptic, anti-catarrhal, and spasmolytic properties $^{(18)}$.

To the best of our knowledge, there are no published data about the anti-QS activity of $C$. nobile extract on $P$. aeruginosa. However, anti-streptococcal and antioxidant activities of essential oils of $C$. nobile have been reported ${ }^{(19)}$.

Recently, several potential quorum sensing inhibitory (QSI) have been discovered from various resources ${ }^{(20)}$. Curcuma longa produces curcumin, which inhibits the expression of virulence genes of $P$. aeruginosa PAO1 ${ }^{(21)}$. 
Furthermore, extracts from different plant parts such as the leaves, flowers, fruit, and bark of Combretum albiflorum, Laurus nobilis and Sonchus oleraceus were also found to possess antiQS activities ${ }^{(22)(23)}$.

Vattem et al. investigated the anti-QS properties of several fruits, plants, and spices at a concentration of $0.5 \mathrm{mg} / \mathrm{ml}$ in the US in 2007. Their results indicated that among all of the extracts tested, only raspberry, blueberry, and grape extracts inhibited AHL activity-mediated violacein production by $60 \%$, $42 \%$, and $20 \%$, respectively. Furthermore, kale, basil, and turmeric decreased PAO1 swarming by $41 \%, 35 \%$, and $32 \%$, respectively ${ }^{(24)}$.

In 2012, Packiavathy et al. ${ }^{(25)}$ investigated the anti-biofilm and anti-QS properties of cumin on gram-negative bacteria such as $P$. aeruginosa and Chromobacterium violaceum. They found that violacein production was inhibited by $2 \mathrm{mg} / \mathrm{ml}$ of the cumin extract, and AHL and biofilm formation were also reduced ${ }^{(25)}$. Although we used a different extract, these results collectively indicate that plant extracts inhibit QS.

In 2011, Alipour et al. ${ }^{(26)}$ investigated the inhibitory effects of an aqueous extract of the roots of American ginseng (NAGE) on QS, twitching, and biofilm formation of $P$. aeruginosa in Canada, and found a MIC and biofilm inhibitory concentration of $1.25-1.5 \%(\mathrm{w} / \mathrm{v})(12.5-25 \mathrm{mg} / \mathrm{ml})$ and $5 \%(\mathrm{w} / \mathrm{v})(50 \mathrm{mg} / \mathrm{ml})$, respectively. Therefore, the MIC concentration was similar to that observed in the present study, but the QS inhibitory concentration differed between NAGE and chamomile extracts $^{(26)}$.

Furthermore, In 2011, Wu et al.(27) also investigated the inhibitory effects of NAGE on biofilm formation by $P$. aeruginosa and found that $5-20 \mathrm{mg} / \mathrm{ml}$ of NAGE extract inhibited biofilm formation but not bacterial growth ${ }^{(27)}$. These results are concordant with the Alipour et al. ${ }^{(26)}$ study with NAGE, but differ to our study in chamomile, suggesting that the difference is due to the specific extract tested. Moreover, it seems that the inhibitory effect of $C$. nobile extract on QS is greater and more efficient than that of NAGE. In our study, the MBC value was very close to the MIC value, which is considered to be a positive feature for any antimicrobial agent.

The formation of a biofilm from agents causing infections such as a wound increases the difficulty of eradication through conventional antibiotic therapy. In this case, C. nobile was able to effectively inhibit the biofilm formation of strains isolated from wound infections, showing its potential value as an alternative anti-infection agent.

Chamomile is a potential source of anti-QS compounds for the development of effective alternative therapeutics. The findings of this study confirm the anti-QS activity of $C$. nobile in $P$. aeruginosa, and suggest that further research at the molecular level is warranted to explore the exact mechanisms of the action of these phytocompounds. The anti-QS property of chamomile may play an important role in antibacterial activity, and offers an additional strategy in the fight against bacterial infections. Indeed, the anti-bacterial concentration was 2-fold greater than the effective concentrations against biofilm formation.

\section{CONFLICT OF INTEREST}

The authors declare that there is no conflict of interest.

\section{REFERENCES}

1. Fereydouni M, Etemadi L, Borouk A. Analgesic effect of flower and leaf extracts of tanacetum parthenium using formalin test in mice. Physiol Pharmmacol 2001; 5:189-198

2. Jaymand K, Rezaei M. A study on chemical composition of essential oils of matricaria chamomilla 1. from Tehran, Hammadan and Kazeroon. Iranian J Med Aromatic Plants 2002; 13:10-24.

3. Nourizadeh E, Mirzapour T, Gasemi K, Rezai M, Latifi NS. Survey of anti-bacterial effects of spearmint, liquorice, perrenial weed, mayweed and thyme on Helicobacter pylori. Daneshvar Medicine 2004; 11:67-71.

4. Honarvaran F, Kesmati M, Esmaeili MH, Jahani Hashemi H, Jafari H, Abbasi E. Effect of chronic administration of Matricaria Chamomilla extract on morphine withdrawal syndrome in adult male mice. Daneshvar Medicine 2007; 14: 27-32.

5. Fuqua WC, Winans SC, Greenberg EP. Quorum sensing in bacteria: the LuxR-LuxI family of cell density-responsive transcriptional regulators. J Bacteriol 1994; 176:269-275.

6. Eberl L. N-acyl homoserinelactone-mediated gene regulation in gram-negative bacteria. Syst Appl Microbiol 1999; 22:493-506.

7. Kleerebezem M, Quadri LE, Kuipers OP, de Vos WM. Quorum sensing by peptide pheromones and two component signal transduction systems in Gram positive bacteria. Mol Microbiol 1997; 24:895-904.

8. Hentzer M, Riedel K, Rasmussen TB, Heydorn A, Andersen JB, Parsek MR, et al. Inhibition of quorum sensing in Pseudomonas aeruginosa biofilm bacteria by a halogenated furanone compound. Microbiology 2002; 148:87-102.

9. Davies DG, Parsek MR, Pearson JP, Iglewski BH, Costerton JW, Greenberg EP. The involvement of cell-to-cell signals in the development of a bacterial biofilm. Science 1998; 280:295-298.

10. Høiby N. Prospects for the prevention and control of pseudomonal infection in children with cystic fibrosis. Paediatr Drugs 2000; 2:451-463.

11. Scutera S, Zucca M, Savoia D. Novel approaches for the design and discovery of quorum-sensing inhibitors. Expert Opin Drug Discov 2014; 9:353-366

12. Husain FM, Ahmad I. Doxycycline interferes with quorum sensing-mediated virulence factors and biofilm formation in Gramnegative bacteria. World J Microbiol Biotechnol 2013; 29:949-957.

13. Berahou A, Auhmani A, Fdil N, Benharref A, Jana M, Gadhi CA. Antibacterial activity of Quercus ilex bark's extracts. J Ethnopharmacol 2007; 112:426-429.

14. French GL. Bactericidal agents in the treatment of MRSA infections--the potential role of daptomycin. J Antimicrob Chemother 2006; 58:1107-1117.

15. Mowat E, Butcher J, Lang S, Williams C, Ramage G. Development of a simple model for studying the effects of antifungal agents on multicellular communities of Aspergillus fumigatus. J Med Microbiol 2007; 56:1205-1212.

16. O'May C, Tufenkji N. The swarming motility of Pseudomonas aeruginosa is blocked by cranberry proanthocyanidins and other tannin-containing materials. Appl Environ Microbiol 2011; 77: 3061-3067. 
17. Vasavi HS, Arun AB, Rekha PD. Anti-quorum sensing activity of Psidium guajava L. flavonoids against Chromobacterium violaceum and Pseudomonas aeruginosa PAO1. Microbiol Immunol 2014; 58:286-293.

18. Sharafzadeh S, Bazrafshan F, Bayatpoor N. Effect of naphthaleneacetic acid and spermidine on essential oil Constituents of German Chamomile. Intl J Agri Crop Sci 2012; 4:1803-1806.

19. Owlia P, Rasooli I, Saderi H. Antistreptococcal and antioxidant activity of essential oil from Matricaria chamomilla L. Res J Biol Sci 2007; 2:237-239.

20. Kalia VC. Quorum sensing inhibitors: an overview. Biotechnol Adv 2013; 31:224-245.

21. Rudrappa T, Bais HP. Curcumin, a known phenolic from Curcuma longa, attenuates the virulence of Pseudomonas aeruginosa PAO1 in whole plant and animal pathogenicity models. J Agric Food Chem 2008; 56:1955-1962.

22. Al-Hussaini R, Mahasneh AM. Microbial growth and quorum sensing antagonist activities of herbal plants extracts. Molecules 2009; 14:3425-3435.
23. Schaefer AL, Greenberg EP, Oliver CM, Oda Y, Huang JJ, BittanBanin $\mathrm{G}$, et al. A new class of homoserine lactone quorum-sensing signals. Nature 2008; 454:595-599.

24. Vattem D, Mihalik K, Crixell SH, McLean RJ. Dietary phytochemicals as quorum sensing inhibitors. Fitoterapia 2007; 78:302-310.

25. Sybiya Vasantha Packiavathy IA, Agilandeswari P, Musthafa KS, Pandian SK, Ravi AV. Antibiofilm and quorum sensing inhibitory potential of Cuminum cyminum and its secondary metabolite methyl eugenol against Gram negative bacterial pathogens. Food Res Int 2012 45:85-92.

26. Alipour M, Omri A, Suntres ZE. Ginseng aqueous extract attenuates the production of virulence factors, stimulates twitching and adhesion, and eradicates biofilms of Pseudomonas aeruginosa. Can J Physiol Pharmacol 2011; 89: 419-427.

27. Wu H, Lee B, Yang L, Wang H, Givskov M, Molin S, et al. Effects of ginseng on Pseudomonas aeruginosa motility and biofilm formation. FEMS Immunol Med Microbiol 2011; 62:49-56. 